\title{
MORPHODYNAMIC UPSCALING WITH THE MORFAC APPROACH
}

\author{
Roshanka Ranasinghe ${ }^{1,2}$, Cilia Swinkels ${ }^{3}$, Arjen Luijendijk ${ }^{3,2}$, Judith Bosboom², Dano \\ Roelvink $^{1,3}$, Marcel Stive ${ }^{2}$ and DirkJan Walstra ${ }^{3,2}$
}

\begin{abstract}
The Morphological Acceleration Factor (MORFAC) approach for morphodynamic upscaling enables the simulation of long term coastal evolution. However the general validity of the MORFAC concept for coastal applications has not yet been comprehensively investigated. Furthermore, a robust and objective method for the a priori determination of the highest MORFAC that is suitable for a given simulation (i.e. critical MORFAC) does not currently exist. This paper presents some initial results of an ongoing, long-term study that attempts to rigorously and methodically investigate the limitations and strengths of the MORFAC approach. Based on the results of a numerical modelling exercise using the morphodynamic model Delft3D, the main dependencies and sensitivities of the MORFAC approach are investigated. Also, a criterion is proposed for the a priori determination of the critical MORFAC, based on the CFL condition for bed form migration.
\end{abstract}

Keywords: morphodynamic modelling; MORFAC; Delft3D; long term coastal evolution

\section{INTRODUCTION}

Until recently it was only possible to numerically simulate coastal evolution at time scales of up to a couple of years while using traditional morphodynamic upscaling techniques such as the "continuity correction' method. The introduction of the morphological acceleration factor (MORFAC) concept to coastal morphodynamic modelling by Lesser et al. (2004) and Roelvink (2006) has changed this. The MORFAC approach enables numerical simulations of coastal morphological evolution due to waves and currents at time scales of decades (Lesser 2009, Tonnon et al. 2007, Jones et al. 2007, Lesser et al. 2004) and - under very uniform forcing conditions (e.g. tides only) - for centuries (Dissanayake et al. 2009a, b, Van der Wegen and Roelvink 2008, Van der Wegen et al. 2008).

Bed level update in coastal morphodynamic models is facilitated via the sediment continuity equation. However as the time scales associated with bed level changes are generally much greater than those associated with hydrodynamic forcing, to enable reasonably fast computations, these models have until recently adopted the approach of updating bed levels and feeding them back into the hydrodynamic calculations only every few hydrodynamic time steps. The MORFAC approach departs from this traditional way of thinking and essentially multiplies the bed levels computed after each hydrodynamic time step by a factor (MORFAC) to enable much faster computation. The significantly upscaled new bathymetry is then used in the next hydrodynamic step, see Fig. 1.

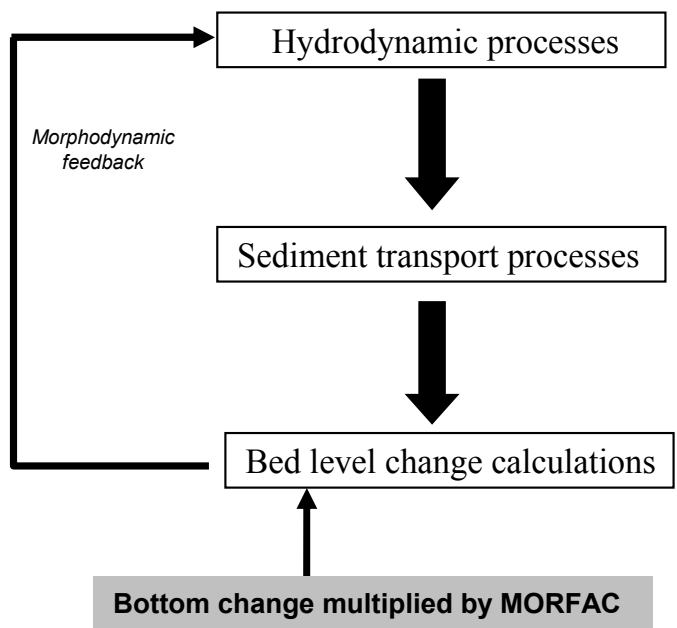

Figure 1. General structure of coastal morphodynamic models and the MORFAC concept.

\footnotetext{
${ }^{1}$ Department of Water Engineering, UNESCO-IHE, PO Box 3015, 2601 DA Delft, The Netherlands

${ }_{2}^{2}$ Civil Engineering and Geosciences, Technical University of Delft, PO Box 5048, 2600 GA Delft, The Netherlands

${ }^{3}$ Harbour, Coastal and Offshore Engineering, Deltares, PO Box 177, 2600 MH Delft, The Netherlands
} 
Although it is very tempting to simply accept the MORFAC concept due to the massive increase in modelling time scales it affords, such a boldly new concept should be rigorously and methodically assessed prior to its general acceptance. This paper presents some initial results of an ongoing study that attempts to systematically investigate the limitations and strengths of the MORFAC approach. Based on the results of a numerical modelling exercise using Delft3D, some of the main dependencies and sensitivities of the MORFAC approach are demonstrated, and a preliminary method for the a priori determination of the critical MORFAC is suggested.

\section{METHODS}

In this study it is assumed that the most accurate numerical model simulation currently possible is one undertaken with MORFAC $(M F)=1$ (i.e. benchmark simulation). Two simple and opposing idealised cases were considered: the morphological evolution of a symmetrical protrusion (hump) and a depression (trench), one being the direct opposite of the other, both morphologically and hydrodynamically. The hump is expected to represent features such as sand bars, while the trench represents channel-like features; both commonly found features in the coastal zone. In both cases, the bed perturbation was initially located on a flat bed and subjected to uniform unidirectional flow. The ambient water depth was set to $4 \mathrm{~m}$ above the plane bed, while the amplitude of the bed perturbation was set to $2 \mathrm{~m}$, resulting in a minimum water depth of $2 \mathrm{~m}$ above the hump, and a maximum water depth of $6 \mathrm{~m}$ in the trench, see Fig. 2.
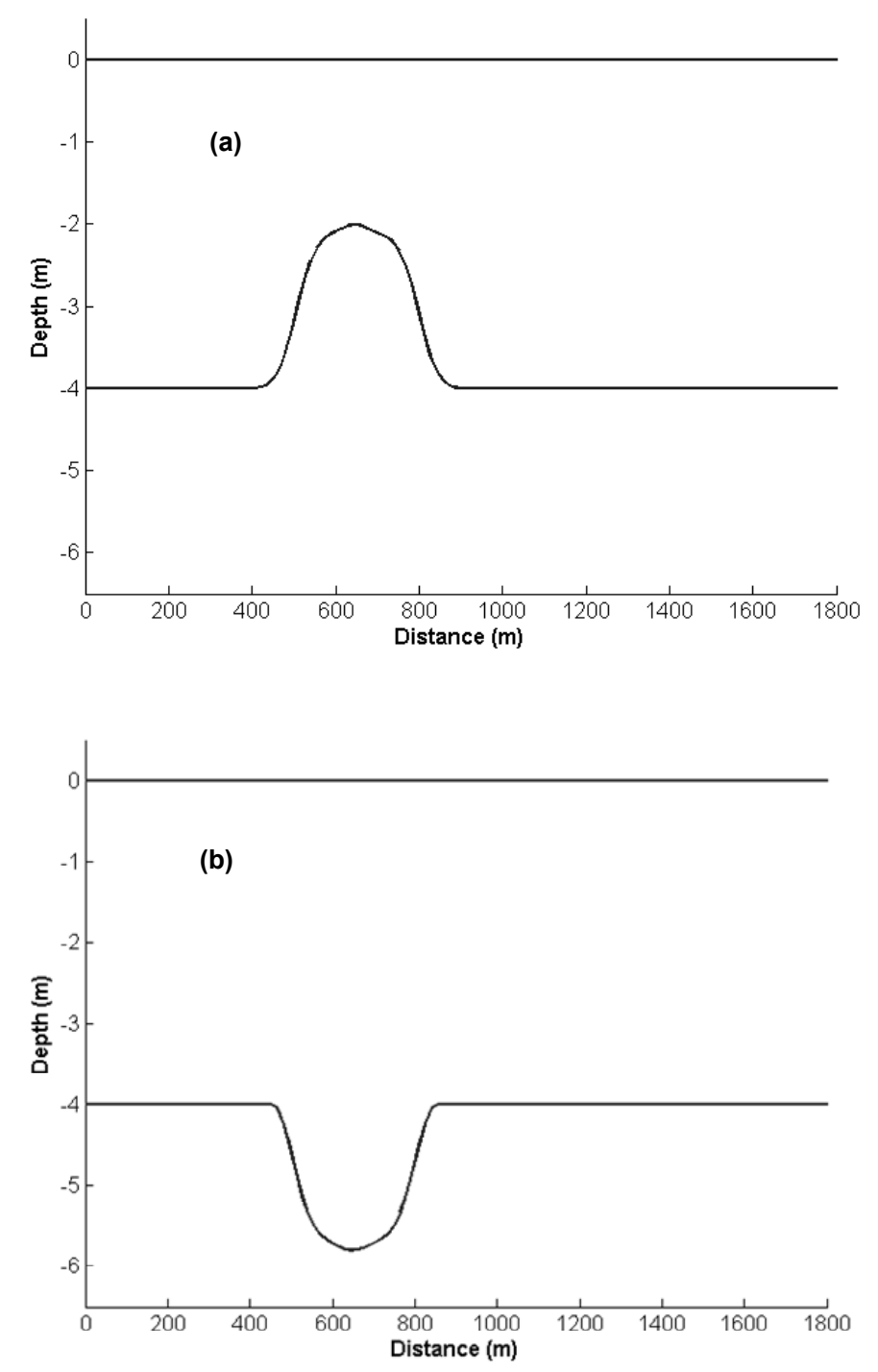

Figure 2. Initial bathymetries: (a) hump on a flat bed, and (b) trench on a flat bed. 
Two series of Delft3D simulations were undertaken in profile mode for the two bed perturbations considered. Uniform unidirectional flow was introduced from the left hand side (LHS) boundary and a zero-gradient flow boundary condition was imposed at the downstream (RHS) boundary. The Engelund and Hansen (1967) total load formulation was used for sediment transport calculations. In each series of simulations, the flow velocity, grid size, hydrodynamic time step and the MORFAC were varied systematically.

The critical MORFAC $\left(M F_{c r i t}\right)$ is the highest MORFAC resulting in bed level predictions that are similar to those predicted by a benchmark case at the same morphological time (MT). Therefore, first, the benchmark conditions have to be determined. The benchmark conditions for each forcing condition were thus obtained by continuing the respective $M F=1$ cases until morphodynamic equilibrium was reached (say at $M T=T_{e}$ ). Morphodynamic equilibrium was defined when both the change in amplitude and the change in propagation speed of the bed perturbation approached zero. The bed levels predicted at $M T=T_{e}$ by subsequent $M F>1$ simulations were then compared with the equilibrium morphology predicted by the benchmark simulation using the Brier Skill Score (BSS) (Van Rijn et al. 2003).

A $B S S$ value of 1 indicates a perfect match between the bed levels predicted by the benchmark case and a case with a higher MORFAC. BSS values lower than unity indicate a difference between bed levels predicted by the two cases; the lower the BSS the greater the divergence between the predicted bed levels. In this study, BSS values less than 0.99 were considered to represent an unacceptable departure from the benchmark case. For a given set of model conditions (flow velocity, grid size, and time step), as the MORFAC was gradually increased from unity, the first MORFAC at which the $B S S$ dropped below 0.99 , before reaching $M T=T_{e}$, was considered as $M F_{\text {crit }}$.

\section{DEPENDENCIES AND SENSITIVITIES}

\section{Hump versus Trench}

Two separate sets of simulations were undertaken for the hump and trench cases with flow velocities $(U)$ of $0.5,0.7,0.9,1.1,1.3$ and $1.5 \mathrm{~m} / \mathrm{s}$. The grid size and hydrodynamic time step were kept constant at $15 \mathrm{~m}$ and $3 \mathrm{~s}$ respectively (resulting in a constant Courant number of 2.5 ) while the MORFAC was systematically increased from 1 to $M F_{\text {crit }}$. The dependency of $M F_{\text {crit }}$ on the ambient Froude number $(F r)$ (i.e. $F r$ at the upstream boundary) is shown in Fig. 3. Two phenomena are clearly visible in Fig. 3. First, for both cases, $M F_{\text {crit }}$ decreases exponentially with ambient $F r$. This is intuitively correct as higher velocities will result in higher sediment transport and thus larger bed level variations which will eventually lead to hydrodynamic instabilities in the model. Second, for a given Froude number, the trench case can accommodate a significantly higher $M F_{\text {crit }}$. This is also intuitively correct as velocities (and thus sediment transport) will increase over the hump while they will decrease over the trench. Therefore, further analysis is restricted to the hump case.

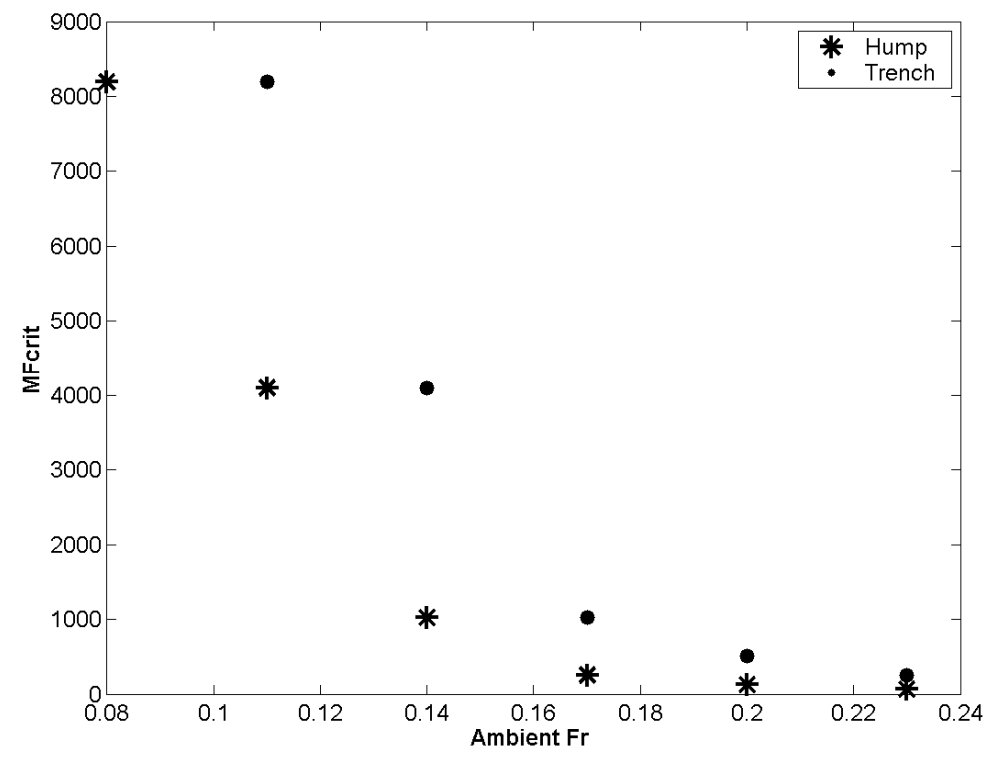

Figure 3. $M F_{\text {crit }}$ versus ambient Froude number for the hump and trench cases. Grid size $=15 \mathrm{~m}$ and hydrodynamic time step $=3 \mathrm{~s}$ in all cases. 
$M F_{\text {crit }}$ versus grid size, hydrodynamic time step and Courant number

A second series of simulations was undertaken to investigate the dependency and sensitivity of $M F_{c r i t}$ on grid size $d x$, hydrodynamic time step $d t$ and Courant number $C r$ for the hump case. These simulations were undertaken for flow velocities $(U)$ of $0.9 \mathrm{~m} / \mathrm{s}$ and $1.3 \mathrm{~m} / \mathrm{s}$. For each $U$, first $d t$ was kept constant at $3 \mathrm{~s}$ while $d x$ was systematically doubled from $3.75 \mathrm{~m}$ to $60 \mathrm{~m}(\sim 1 / 5$ th of hump width). Then $d x$ was kept constant at $15 \mathrm{~m}$ while $d t$ was systematically doubled from $0.75 \mathrm{~s}$ to $12 \mathrm{~s}$. This set of simulations resulted in $\mathrm{Cr}$ values that varied between 0.5 and 10 (10 being the largest recommended $\mathrm{Cr}$ value for Delft3D). $M F_{c r i t}$ vs. $C r$ for this set of simulations is plotted in Fig. 4 which shows that $M F_{c r i t}$ can vary by up to 1500 for the same $C r$. Thus $M F_{c r i t}$ appears not to be directly governed by $C r$.

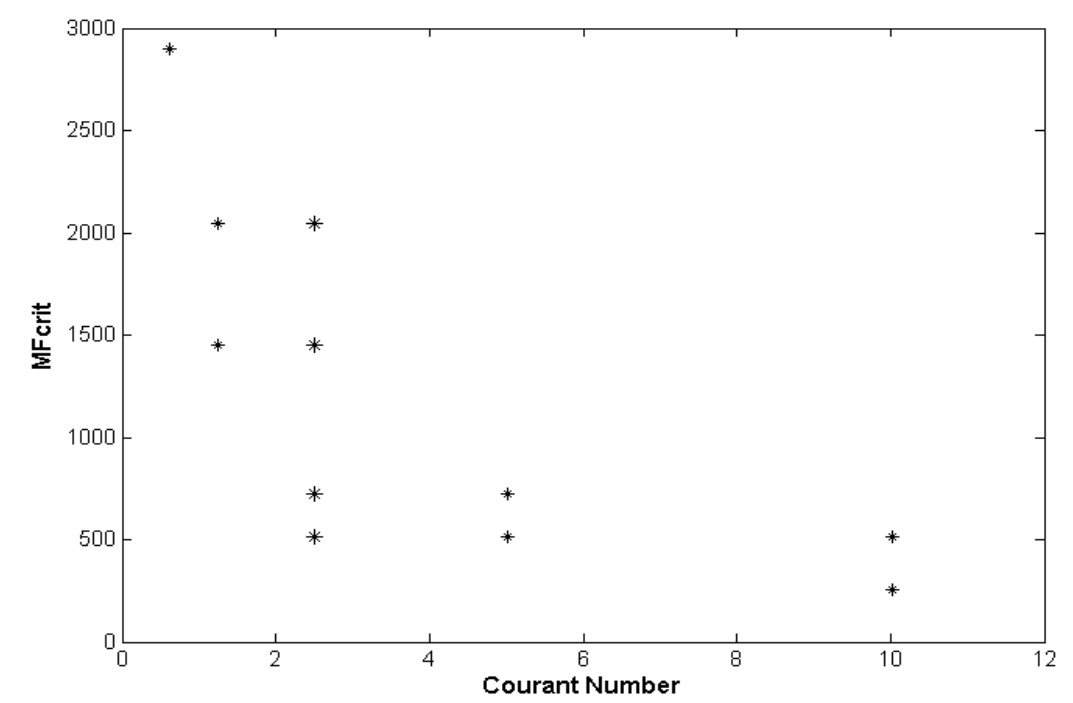

Figure 4. $M F_{c r i t}$ versus $C r$ for $U=0.9 \mathrm{~m} / \mathrm{s}$. $d x$ was varied between $3.75 \mathrm{~m}$ and $60 \mathrm{~m}$ while $d t$ was varied between 0.75 s and $12 \mathrm{~s}$. Cr varied freely between 0.5 and 10.

To investigate the individual dependencies between $M F_{c r i t}$ and $d x, d t$, in addition to the above set of simulations where $C r$ was allowed to freely vary while $d x$ and $d t$ were varied, another set of simulations where $C r$ was forced to remain constant at 2.5 while $d x$ and $d t$ were varied was undertaken. Fig. 5 shows the resulting variations of $M F_{c r i t}$ with $d x$ and $d t$ for both constant and varying $C r$.

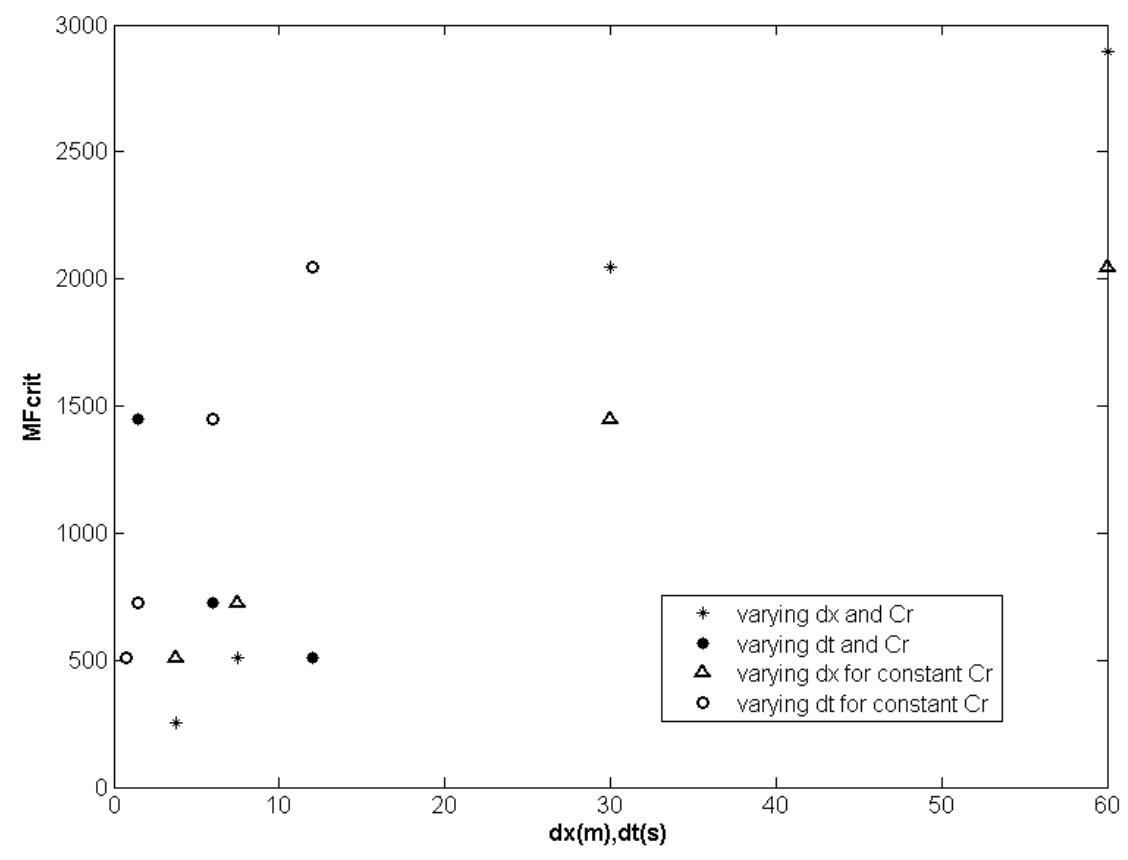

Figure 5. $M F_{\text {crit }}$ versus grid size $(d x)$, hydrodynamic time step $(d t)$ and Courant number $(C r)$. 
An almost linear correlation between $M F_{c r i t}$ and $d x$ can be seen, regardless of whether $C r$ is constant or varying. However, the dependency of $M F_{c r i t}$ on $d t$ is less clear. For the cases where $C r$ was allowed to vary with $d t$ (i.e. by keeping $d x$ constant - solid circles), $M F_{c r i t}$ increases with decreasing $d t$, which is intuitively correct as numerical stability increases with decreasing $d t$. However, when $C r$ is forced to be constant while increasing/decreasing $d t$ (i.e. by simultaneously decreasing/increasing $d x$ ), $M F_{c r i t}$ decreases with decreasing $d t$ (unfilled circles), which is counter-intuitive. This indicates that the dependency of $M F_{c r i t}$ on $d x$ over-rides that on $d t$.

\section{CRITERION FOR THE A PRIORI DETERMINATION OF $\boldsymbol{M F}_{\text {crit }}$}

To ensure numerical stability, the propagation of bed forms in one morphological time step should not exceed the grid cell size (CFL criterion). The propagation speed (or celerity) of bed forms $C_{b e d}$ is commonly estimated as:

$$
C_{\text {bed }}=\frac{b|\vec{S}|}{(1-\varepsilon) h}
$$

where $b$ is the power of the sediment transport formulation used, $\varepsilon$ is the porosity, $h$ is the water depth and $|\vec{S}|$ is the sediment transport magnitude.

Including the effect of MORFAC, the above mentioned $C F L$ condition requires that:

$$
C F L_{M F}=\frac{C_{b e d} M F d t}{d x}<1
$$

To examine whether the simulations undertaken here satisfy the above criterion, the $C F L_{M F}$ values for the last successful simulation (i.e. the simulation associated with $M F_{c r i t}$ ) and the first unsuccessful simulation for each simulated combination of $U, d x$ and $d t$ are plotted as a binary plot (see Fig. 6). Successful and unsuccessful simulations are indicated by values of 1 and -1 respectively on the Y-axis of Fig. 6.

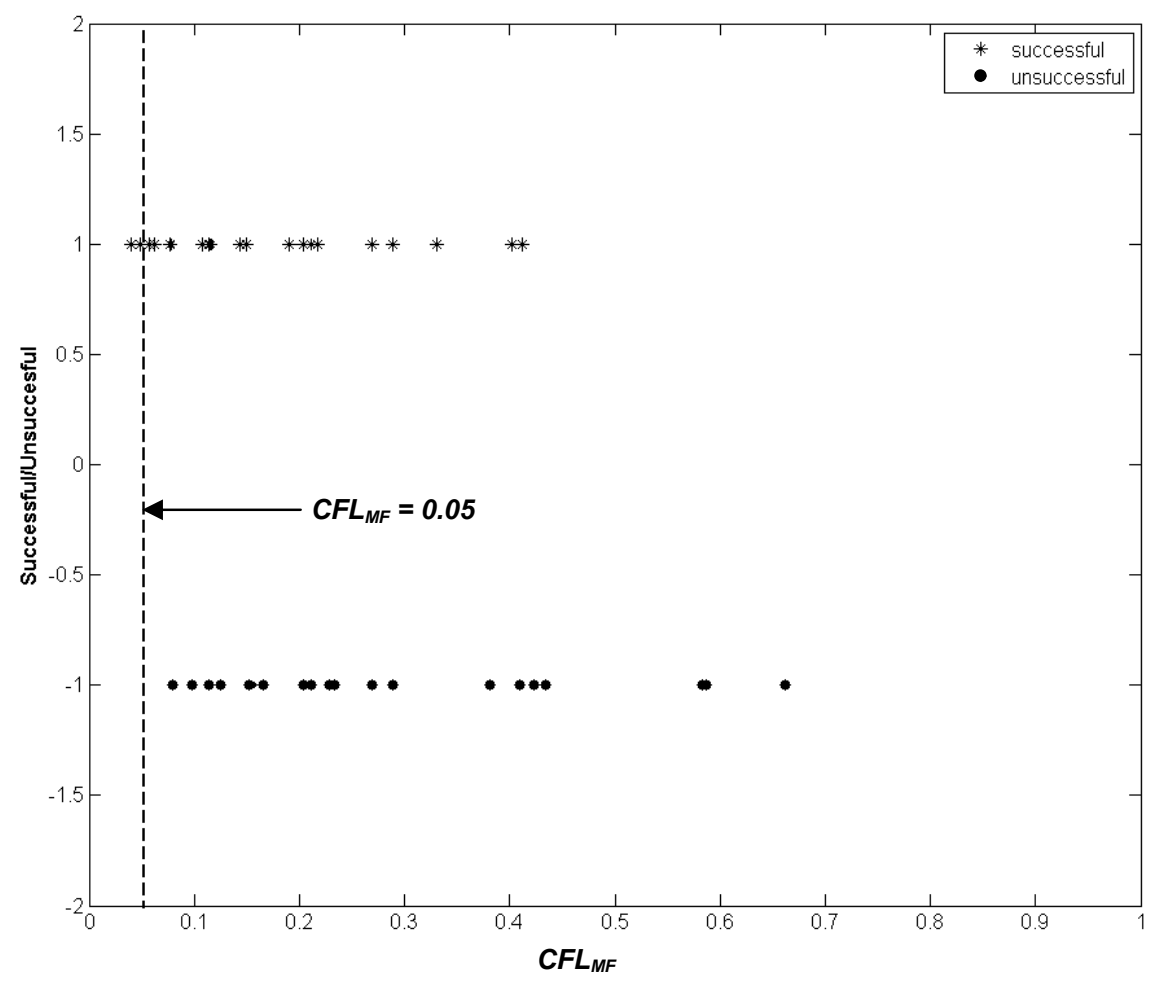

Figure 6. $C F L$ for bed form propagation $\left(C F L_{M F}\right)$ for the last successful simulation (i.e. the simulation associated with $M F_{\text {crit) }}$ (asterisks) and the first unsuccessful simulation (solid circles) for each simulated combination of $U, d x$ and $d t$. 
It is clear that the $C F L_{M F}$ values associated with both successful and unsuccessful simulations fall below the critical value of 1 . This indicates that while the $M F_{\text {crit }}$ simulations do satisfy the $C F L_{M F}$ criterion given by Eq. 2, simulations fail at lower $M F$ values than they should. Otherwise, all the circles (i.e. unsuccessful cases) in Fig. 6 would have been located farther to the right on the X-axis such that the associated $C F L_{M F}$ values would be $>1$. There could be numerous reasons for this premature failure including: failure of the assumed linear relationship between morphological response and hydrodynamic response; MORFAC induced errors in bed form celerity and/or amplitude; and variable relationship between MORFAC and numerical errors (due to advection/diffusion scheme), et cetera.

Based on the above analysis, it is clear that the development of a simple and definitive criterion to predict $M F_{\text {crit }}$ for even this simplest case of unidirectional flow over a symmetric bed feature is nontrivial. Nevertheless, based on Fig. 6, it appears that, at least for the range of conditions tested here, $C F L_{M F}<0.05$ may be used as a preliminary guide to obtain a safe first estimate of $M F_{c r i t}$. This returns a $M F_{\text {crit }}$ value of about 100 when typical values used in nearshore coastal applications are substituted in Eq. 2 for $d t(3-10 \mathrm{~s}), d x(5-20 \mathrm{~m})$ and $C_{b e d}(\sim 0.001 \mathrm{~m} / \mathrm{s})$.

\section{CONCLUSIONS}

A strategically designed series of Delft3D simulations has provided new insights regarding the dependencies and sensitivities of the MORFAC approach for morphodynamic upscaling. The main findings are:

1. The critical MORFAC $\left(M F_{c r i t}\right)$, has a strong dependency on the Froude number $F r$ and the grid size $d x . M F_{\text {crit }}$ decreases exponentially as $F r$ increases, while it increases almost linearly with $d x$.

2. $M F_{c r i t}$ does not appear to be directly governed by the Courant number $(\mathrm{Cr})$.

3. The criterion $C F L_{M F}<0.05$ may provide a safe first estimate of $M F_{\text {crit }}$.

It should be noted that the results and conclusions presented herein may not be directly applicable to complex real-life situations which are likely to incorporate highly non-uniform morphology and time varying non-linear forcing which may include tides, waves, wind etc. Research is currently being undertaken to further investigate the many dependencies and sensitivities of the MORFAC approach with the ultimate goal of developing an effective criterion for the a priori determination of $M F_{\text {crit }}$ for any given real-life situation.

\section{REFERENCES}

Engelund, F. and E. Hansen. 1967. A monograph on Sediment Transport in Alluvial Streams. Teknisk Forlag, Copenhagen, Denmark.

Jones, O. P., O.S. Petersen, and H. Kofoed-Hansen. 2007. Modelling of complex coastal environments: Some considerations for best practice. Coastal Engineering, 54: 717-733.

Lesser, G. 2009. An approach to medium-term coastal morphological modeling, PhD-thesis. Delft University of Technology, ISBN 978-0-415-55668-2.

Lesser, G., J.A. Roelvink, J.A.T.M. Van Kester, and G.S. Stelling. 2004. Development and validation of a three-dimensional morphological model. Coastal Engineering, 51: 883-915.

Roelvink, J.A., 2006. Coastal morphodynamic evolution techniques. Coastal Engineering, 53: 277287.

Tonnon, P.K., L.C. Van Rijn, and D.J.R. Walstra, D. J. R. 2007. Morphodynamic modelling of tidal sand waves on the shoreface. Coastal Engineering, 54: 279-296.

Dissanayake, D.M.P.K., R. Ranasinghe, and J.A. Roelvink. 2009. Effect of sea level rise on tidal inlet evolution, J. Coastal Research, SI 56(2): 942-946.

Dissanayake, D.M.P.K., J.A. Roelvink, and M. Van der Wegen. 2009. Modelled channel patterns in a schematized tidal inlet. Coastal Engineering, 56: 1069-1083.

Van der Wegen, M., and J.A. Roelvink. 2008. Long-term morphodynamic evolution of a tidal embayment using a two-dimensional, process-based model. J. Geophysical Research, 113, C03016. doi:10.1029/2006JC003983.

Van der Wegen, M., Z.B. Wang, H.H.G. Savenije, and J.A. Roelvink. 2008. Long-term morphodynamic evolution and energy dissipation in a coastal plain, tidal embayment, $J$. Geophysical. Research, 113, F03001, doi:10.1029/2007JF000898. 
Van Rijn, L.C., D.J.R. Walstra, B. Grasmeijer, J. Sutherland, S. Pan, and J.P. Sierra. 2003. The predictability of cross-shore bed evolution of sandy beaches at the time scale of storms and seasons using process-based profile models. Coastal Engineering, 47: 295-327. 